\title{
Diversity-Multiplexing Tradeoff in MIMO Relay Channels for a Broad Class of Fading Distributions
}

\author{
Sergey Loyka, Senior Member, IEEE, and Georgy Levin
}

\begin{abstract}
Diversity multiplexing tradeoff (DMT) in MIMO relay channels, where the transmitter and receiver are equipped with multiple antennas and the relay with a single one, is derived for a broad class of fading distributions, including, as special cases, non-identical, spatially correlated, and non-zero mean channels. It is shown that the DMT does not depend on a particular fading distribution, but rather on its polynomial behavior near zero. The DMT turns out to be the same for the simple "amplify-and-forward" mode and more complicated "decode-and-forward" relaying (with capacity achieving codes), i.e. the full processing capability at the relay does not help to improve the DMT.
\end{abstract}

Index Terms-Multi-antenna (MIMO) system, diversity multiplexing trade-off.

\section{INTRODUCTION}

$\mathbf{T}$ O overcome difficulties in performance analysis of MIMO systems, an elegant framework termed "diversitymultiplexing tradeoff" (DMT) has been proposed in [1] that allows one to quantify asymptotically the MIMO system performance in terms of two principle gains it provides, diversity and multiplexing, available in a fading channel when SNR $\rightarrow \infty$ [2]. While the original DMT formulation of Zheng and Tse [1] is limited to i.i.d. Rayleigh fading channels, a generalization to a class of channels satisfying a number of conditions on the distribution function has been presented in [3]. It has been shown that full-rank correlation does not affect the DMT and that it is the same in the Rayleigh and Ricean channels. Inspired by the DMT framework, a number of spacetime coding techniques that achieve the diversity-multiplexing tradeoff have been proposed, see e.g. [4].

The emerge of cooperative communication strategies, which exploit multi-user diversity available in wireless networks, has inspired the study of diversity-multiplexing tradeoff in relay channels. While the research in that area was initially concentrated on the single-antenna systems [5][6], the emphasis has recently shifted towards multi-antenna systems [7]-[9]. Since the MIMO systems present an additional level of difficulty in terms of performance evaluation, many relay systems, for which the outage probability/capacity analysis is not available in a manageable closed form, have been characterized and compared via their respective DMTs. Most of the studies on relay channels employ the Rayleigh or Ricean fading model with independent links [7]-[10]. The only exception is [11], where the outage probability/capacity has been studied for a

Manuscript received October 9, 2009. The associate editor coordinating the review of this letter and approving it for publication was R. Nabar.

The authors are with the School of Information Technology and Engineering, University of Ottawa, Ontario, Canada, K1N 6N5 (e-mail: sergey.loyka@ieee.org, glevin@site.uottawa.ca).

Digital Object Identifier 10.1109/LCOMM.2010.04.091992

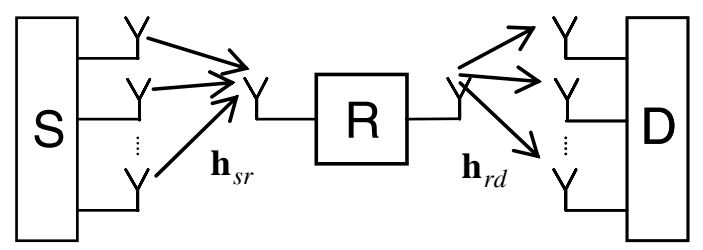

Fig. 1. Relay channel with a single relay node (R) (single-antenna) and multiple-antenna source (S) and destination (D).

generic fading distribution, but the analysis was limited to the low-SNR regime and the links are still required to be independent.

In the present letter, we allow the fading to be non-identical, spatially correlated and of generic distribution (which includes Rayleigh-fading and other popular models as special cases), and consider amplify-and-forward and decode-and-forward protocols. The source and the destination terminals are assumed to be equipped with multiple antennas and the relay node with a single one (e.g. due to complexity constraints).

The contribution of the letter is two-fold:

- the DMT of this relay channel is obtained for a broad class of fading distributions, which behave polynomially near zero (Theorem 1).

- Under this condition, the single-relay channel is shown to have the same DMT in the amplify-and-forward and decodeand-forward modes (Theorem 2), i.e. the full processing capability at the relay does not help to improve the DMT.

\section{Channel Model}

Consider a MIMO relay channel with a single relay node equipped with a single antenna (see Fig. 1); the source and the destination terminals are equipped with multiple antennas. While we consider both the amplify-and-forward and decodeand-forward protocols, the former will be assumed for simplicity of exposition, unless indicated otherwise, with a fixed gain relay (this is motivated by the fact that it is simpler to implement). We assume no direct source-destination link. This is motivated by three reasons: (i) the direct link is usually much weaker than the relay one (e.g. no line-of-sight etc.), and thus can be neglected (this is the case when the relay link is needed most) [6]; (ii) the results obtained for the no direct link case can be used as a "building block" to analyze the direct link case; (iii) the direct link corresponds to the regular (no relay) systems and thus has been extensively studied and is well understood by now, the relay link, on the contrary, is not so well understood so we concentrate our attention on this case. 
The standard baseband system model of a frequency flat relay channel in the amplify-and-forward mode is,

$$
\mathbf{y}=\sqrt{K_{r} G_{r d} G_{s r}} \mathbf{h}_{r d} \mathbf{h}_{s r}^{+} \mathbf{x}+\sqrt{K_{r} G_{r d}} \mathbf{h}_{r d} \xi_{r}+\boldsymbol{\xi}
$$

where $\mathbf{x}$ and $\mathbf{y}$ are the source (transmitter) and destination (receiver) symbol vectors, $\mathbf{h}_{s r}$ and $\mathbf{h}_{r d}$ are the source-relay and relay-destination normalized channels (i.e. they include the multipath fading but not the average path loss), ${ }^{+}$denotes Hermitian conjugation, $G_{s r}$ and $G_{r d}$ are the source-relay and relay-destination average path loss factors, $\xi_{r} \sim \mathcal{C N}\left(0, \sigma_{r}^{2}\right)$ and $\boldsymbol{\xi} \sim \mathcal{C N}\left(\mathbf{0}, \sigma_{0}^{2} \mathbf{I}\right)$ are relay and destination AWGN noise of variance $\sigma_{r}^{2}$ and $\sigma_{0}^{2}$ respectively, and independent of each other. Without loss of generality, the relay gain $K_{r}$ can be further absorbed in $G_{r d}$ via the substitution $K_{r} G_{r d} \rightarrow G_{r d}$. We do not assume any particular fading distribution, but only assume that $\mathbf{h}_{s r}$ and $\mathbf{h}_{r d}$ are independent of each other, and require that the PDFs $f_{s}(x)$ and $f_{d}(x)$ of $\mathbf{h}_{s r}$ and $\mathbf{h}_{r d}$ behave polynomially near zero, which hold for a broad class of fading distributions including, as special cases, Rayleigh, Ricean, Nakagami, and Weibull fading, which may be correlated, nonidentical and non-zero mean. Thus, our results hold for a generic fading channel. Note that $1^{\text {st }}$ term in (1) represents the signal received at the destination; $2^{\text {nd }}$ and $3^{\text {rd }}$ terms represent the relay noise propagated to the destination and the destination noise.

The sufficient statistics for $\mathbf{y}$ is [2],

$z=\frac{\mathbf{h}_{r d}^{+}}{\left|\mathbf{h}_{r d}\right|} \mathbf{y}=\sqrt{G_{r d} G_{s r}}\left|\mathbf{h}_{r d}\right| \mathbf{h}_{s r}^{+} \mathbf{x}+\sqrt{G_{r d}}\left|\mathbf{h}_{r d}\right| \xi_{r}+\frac{\mathbf{h}_{r d}^{+}}{\left|\mathbf{h}_{r d}\right|} \boldsymbol{\xi}$

where $|\mathbf{h}|^{2}=\mathbf{h}^{+} \mathbf{h}$, and the instantaneous SNR at the destination can be expressed as

$$
\mathrm{SNR}=\frac{G_{r d} G_{s d}\left|\mathbf{h}_{r d}\right|^{2} \mathbf{h}_{s r}^{+} \mathbf{R}_{x} \mathbf{h}_{s r}}{\sigma_{0}^{2}+G_{r d}\left|\mathbf{h}_{r d}\right|^{2} \sigma_{r}^{2}} \leq \gamma=\frac{\left|\mathbf{h}_{r d}\right|^{2}\left|\mathbf{h}_{s r}\right|^{2}}{1+\alpha\left|\mathbf{h}_{r d}\right|^{2}} \gamma_{0},
$$

where $\mathbf{R}_{x}=E\left\{\mathbf{x x}^{+}\right\}$is the covariance matrix of the transmitted signal, $\alpha=G_{r d} \sigma_{r}^{2} / \sigma_{0}^{2}$ is the ratio of the average relay noise propagated to the destination to the destination noise, and $\gamma_{0}=G_{r d} G_{s r} \sigma_{x}^{2} / \sigma_{0}^{2}$ is the average SNR at the destination, $\sigma_{x}^{2}=\operatorname{tr} \mathbf{R}_{x}=E\left\{\mathbf{x}^{+} \mathbf{x}\right\}$ is the total transmitted power (at the source). The inequality in (3) follows from $\mathbf{h}_{s r}^{+} \mathbf{R}_{x} \mathbf{h}_{s r} \leq\left|\mathbf{h}_{s r}\right|^{2} \sigma_{x}^{2}$, and the equality is achieved when $\mathbf{R}_{x}=\sigma_{x}^{2} \mathbf{h}_{s r} \mathbf{h}_{s r}^{+} /\left|\mathbf{h}_{s r}\right|^{2}$, i.e. beamforming from the source to the relay, $\mathbf{x}=s \cdot \mathbf{h}_{s r} /\left|\mathbf{h}_{s r}\right|$, where $s$ is the scalar transmitted symbol of the total power $\sigma_{x}^{2}$. This requires channel state information (CSI) at the source. When no such information is available, a sensible transmission strategy is isotropic [2], i.e. $\mathbf{R}_{x}=\sigma_{x}^{2} \mathbf{I} / m$, where $m$ is the number of source antennas. In this case, the instantaneous SNR at the destination is $\gamma / m$, i.e. the source CSI brings in an $m$-fold SNR gain, but does not change the statistics of the instantaneous SNR and, therefore, the outage probability or outage capacity differ by a constant SNR shift, and the diversity-multiplexing tradeoff is the same in both cases.

\section{Diversity-MultipleXing TradeOFF}

The diversity and multiplexing gains are defined as [1]

$$
d=-\lim _{\gamma_{0} \rightarrow \infty} \ln P_{\text {out }} / \ln \gamma_{0}
$$

$$
r=\lim _{\gamma_{0} \rightarrow \infty} R / \ln \gamma_{0},
$$

where $P_{\text {out }}(R)=\operatorname{Pr}\{C<R\}$ is outage probability, $R$ is the target rate, and $C=\ln (1+\gamma)$ is the instantaneous channel capacity in $[\mathrm{nat} / \mathrm{s} / \mathrm{Hz}]$, i.e. the capacity given a channel realization.

Definition: Functions $f(x)$ and $g(x)$ are asymptotically equivalent, $f(x) \sim g(x)$, if there exist constants $0<A \leq$ $B<\infty$ such that $A g(x) \leq f(x) \leq B g(x)$ for sufficiently small $x$.

Under this setting, the DMT is given by the following theorem:

Theorem 1: Consider the relay channel in (1) such that the PDFs $f_{s}(x)$ and $f_{d}(x)$ of $\left|\mathbf{h}_{s r}\right|^{2}$ and $\left|\mathbf{h}_{r d}\right|^{2}$ behave polynomially near zero, i.e. $f_{s}(x) \sim x^{d_{s}-1}, f_{d}(x) \sim x^{d_{d}-1}$ as $x \rightarrow 0$, where $d_{s}, d_{d}$ are the diversity gains (orders) of the source-relay and relay-destination links at $r=0$. The DMT of this channel in the amplify-and-forward mode is

$$
\begin{gathered}
d(r)=\min \left(d_{s}(r), d_{d}(r)\right)=\min \left(d_{s}, d_{d}\right)(1-r), \\
0 \leq r \leq 1
\end{gathered}
$$

where $d_{s}(r)=d_{s}(1-r), d_{d}(r)=d_{d}(1-r)$ are the DMTs of the source-relay and relay-destination links.

Proof: see Appendix.

Theorem 1 holds for a broad class of fading distributions in the relay channel, which include, as special cases, Rayleigh, Rice, Nakagami, and Weibull, and which may be spatially correlated and non-identically distributed. It is thus a generalization of [[7], Theorem 1], where $\mathbf{h}_{s r}$ and $\mathbf{h}_{r d}$ were assumed to be i.i.d. complex Gaussian. Note also that the noise at the relay node does not affect the DMT, i.e. the channel in (1) with $\xi_{r}=0$ has the same DMT as in (6).

Theorem 1 demonstrates that the DMT depends on the number of degrees of freedom available in the channel and not on particular fading distribution, as long as the definition of diversity gain in (4) makes sense. Similar result has been also established for full-rank MIMO channels in [3]. Since the conditions of Theorem 1 relate to the behavior near zero of the distributions of $\left|\mathbf{h}_{s r}\right|^{2}$ and $\left|\mathbf{h}_{r d}\right|^{2}$, not the individual vector entries, it is straightforward to see that a full-rank correlation does not change the DMT since the polynomial behavior near zero is not affected. Following the discussion in section II, the source CSI is equivalent to an $m$-fold SNR gain and, therefore, has no effect on the DMT. The transmit beamforming in combination with QAM modulation (e.g. see [2]) is an example of a space-time code that achieves the DMT of the single-relay channel with the source CSI. When no such CSI is available, isotropic transmission in combination with QAM will achieve the DMT.

Let us now consider the DMT of the decode-and-forward single-relay channel, assuming capacity achieving codes and complete decoding/encoding at the relay. The following theorem holds:

Theorem 2: Under the conditions of Theorem 1, the diversity-multiplexing tradeoff of the decode-and-forward single-relay channel is given by (6).

Proof: see Appendix.

Therefore, the single-relay channel subject to fading from a broad class of distributions has the same DMT in the 
amplify-and-forward and decode-and-forward modes, i.e. the full processing capability at the relay (to achieve the capacity) does not help to improve the DMT.

\section{APPENDIX}

Proof of Theorem 1: Let $g_{s}=\left|\mathbf{h}_{s r}\right|^{2}, g_{d}=\frac{\left|\mathbf{h}_{r d}\right|^{2}}{1+\alpha\left|\mathbf{h}_{r d}\right|^{2}}$. From (3) the outage probability is

$$
P_{\text {out }}=\operatorname{Pr}\{C<R\}=\operatorname{Pr}\left\{g_{s} g_{d}<\varepsilon\right\},
$$

where $\varepsilon=\left(e^{R}-1\right) / \gamma_{0}$. For $R=r \ln \gamma_{0}, 0 \leq r<1$, and $\gamma_{0} \rightarrow \infty, \varepsilon \sim \gamma_{0}^{-(1-r)}$, i.e. $\varepsilon \rightarrow 0$ as $\gamma_{0} \rightarrow \infty$. From (7),

$$
P_{\text {out }}=\int_{0}^{\infty} f_{s}\left(g_{s}\right) F_{d}\left(\varepsilon / g_{s}\right) d g_{s}=P_{1}+P_{2}+P_{3},
$$

where $F_{d}(x)$ is the CDF of $g_{d}$, and

$$
\begin{gathered}
P_{1}=\int_{0}^{\varepsilon \ln \gamma_{0}} f_{s}\left(g_{s}\right) F_{d}\left(\varepsilon / g_{s}\right) d g_{s}, \\
P_{2}=\int_{\varepsilon \ln \gamma_{0}}^{\delta} f_{s}\left(g_{s}\right) F_{d}\left(\varepsilon / g_{s}\right) d g_{s} \\
P_{3}=\int_{\delta}^{\infty} f_{s}\left(g_{s}\right) F_{d}\left(\varepsilon / g_{s}\right) d g_{s},
\end{gathered}
$$

and $\varepsilon \ln \gamma_{0}<\delta<<1, \delta$ is a sufficiently small constant (independent of the SNR). In fact, $P_{1}$ and $P_{3}$ represent the outage events due to the outage at the source and destination links, respectively, and $P_{2}$ represents the outage events due to simultaneous deep fades at both links. The upper bound on $P_{1}$ is

$$
P_{1} \leq \int_{0}^{\varepsilon \ln \gamma_{0}} f_{s}\left(g_{s}\right) d g_{s}=F_{s}\left(\varepsilon \ln \gamma_{0}\right)
$$

where $F_{s}(x)$ is the CDF of $g_{s}$. Since $f_{s}(x) \sim x^{d_{s}-1}, F_{s}(x) \sim$ $x^{d_{s}}$, and from (12),

$$
P_{1} \leq F_{s}\left(\varepsilon \ln \gamma_{0}\right) \sim\left(\varepsilon \ln \gamma_{0}\right)^{d_{s}} \doteq \varepsilon^{d_{s}},
$$

where $f_{1}\left(\gamma_{0}\right) \doteq f_{2}\left(\gamma_{0}\right)$ means exponential equality [1],

$$
\lim _{\gamma_{0} \rightarrow \infty} \frac{\ln f_{1}\left(\gamma_{0}\right)}{\ln \gamma_{0}}=\lim _{\gamma_{0} \rightarrow \infty} \frac{\ln f_{2}\left(\gamma_{0}\right)}{\ln \gamma_{0}}
$$

If $f_{1}\left(\gamma_{0}\right) \sim f_{2}\left(\gamma_{0}\right)$ as $\gamma_{0} \rightarrow \infty$, then $f_{1}\left(\gamma_{0}\right) \doteq f_{2}\left(\gamma_{0}\right)$. Note that $F_{d}(x)=F_{r d}\left(\frac{x}{1-\alpha x}\right)$ for $\alpha x \leq 1$ and 1 otherwise, where $F_{r d}(x)$ is the CDF of $\left|\mathbf{h}_{r d}\right|^{2}$. Thus, $F_{d}(x) \sim F_{r d}(x) \sim x^{d_{d}}$ for sufficiently small $x$, since $F_{r d}(x) \sim x^{d_{d}}$. As $\delta$ is sufficiently small,

$$
P_{2} \sim \int_{\varepsilon \ln \gamma_{0}}^{\delta} g_{s}^{d_{s}-1}\left(\varepsilon / g_{s}\right)^{d_{d}} d g_{s}=\varepsilon^{d_{d}} \int_{\varepsilon \ln \gamma_{0}}^{\delta} g_{s}^{d_{s}-d_{d}-1} d g_{s}
$$

If $d_{s} \neq d_{d}$

$$
P_{2} \sim \frac{\varepsilon^{d_{d}}}{d_{d}-d_{s}}\left(\left(\varepsilon \ln \gamma_{0}\right)^{d_{s}-d_{d}}-\delta^{d_{s}-d_{d}}\right) \doteq \varepsilon^{\min \left(d_{s}, d_{d}\right)}
$$

If $d_{s}=d_{d}$,

$$
P_{2} \sim \varepsilon^{d_{s}}\left(\ln \delta-\ln \left(\varepsilon \ln \gamma_{0}\right)\right) \doteq \varepsilon^{d_{s}}
$$

The upper bound on $P_{3}$ is

$$
P_{3} \leq \int_{\delta}^{\infty} f_{s}\left(g_{s}\right) F_{d}(\varepsilon / \delta) d g_{s}=\left(1-F_{s}(\delta)\right) F_{d}(\varepsilon / \delta) \doteq \varepsilon^{d_{d}}
$$

Combining (13), (16), and (18), one obtains

$$
P_{\text {out }} \doteq \varepsilon^{\min \left(d_{s}, d_{d}\right)} \doteq \gamma_{0}^{-\min \left(d_{s}, d_{d}\right)(1-r)}
$$

Applying (4), (6) follows.

Proof of Theorem 2: The instantaneous capacity of the decode-and-forward single-relay channel is

$$
C=\min \left\{C_{s r}, C_{r d}\right\} \text {, }
$$

where $C_{s r}=\ln \left(1+G_{s r}\left|\mathbf{h}_{s r}\right|^{2} \sigma_{x}^{2} / \sigma_{r}^{2}\right)$ and $C_{r d}=$ $\ln \left(1+G_{r d}\left|\mathbf{h}_{r d}\right|^{2} \sigma_{R}^{2} / \sigma_{0}^{2}\right)$ are the capacities of the sourcerelay and relay-destination links, $\sigma_{R}^{2}$ is the relay transmit power. Thus, the outage probability is

$$
P_{\text {out }}=\operatorname{Pr}\{C<R\}=P_{s r}+P_{r d}-P_{s r} P_{r d},
$$

where $P_{s r(r d)}=\operatorname{Pr}\left\{C_{s r(r d)}<R\right\}$ are the outage probabilities of the source-relay and relay-destination links. Under the adopted assumptions $P_{s r} \sim \gamma_{0}^{-d_{s}(1-r)}, P_{r d} \sim \gamma_{0}^{-d_{d}(1-r)}$. Thus, as $\gamma_{0} \rightarrow \infty$

$$
P_{\text {out }} \rightarrow \max \left\{P_{\text {sr }}, P_{r d}\right\} \doteq \gamma_{0}^{-\min \left(d_{s}, d_{d}\right)(1-r)}
$$

Applying (4), (6) follows.

\section{REFERENCES}

[1] L. Zheng and D. N. C. Tse, "Diversity and multiplexing: a fundamental tradeoff in multiple-antenna channels," IEEE Trans. Inf. Theory, vol. 49, no. 5, pp. 1073-1096, May 2003.

[2] D. N. C. Tse and P. Viswanath, Fundamentals of Wireless Communications. Cambridge University Press, 2005.

[3] L. Zhao, W. Mo, Y. Ma, and Z. Wang, "Diversity and multiplexing tradeoff in general fading channels," IEEE Trans. Inf. Theory, vol. 53, no. 4, pp. 1549-1557, Apr. 2007.

[4] P. Elia et al., "Explicit space-time codes achieving the diversitymultiplexing gain tradeoff," IEEE Trans. Inf. Theory, vol. 52, no. 9, pp. 3869-3884, Sep. 2006.

[5] N. Laneman, D. Tse, and G. Wornell, "Cooperative diversity in wireless networks: efficient protocols and outage behavior," IEEE Trans. Inf. Theory, vol. 50, no. 11, pp. 3062-3080, Dec. 2004.

[6] J. Boyer, D. D. Falconer, and H. Yanikomeroglu, "Multihop diversity in wireless relaying channels," IEEE Trans. Commun., vol. 52, no. 10, pp. 1820-1830, Oct. 2004

[7] C. Rao and B. Hassibi, "Diversity-multiplexing gain trade-off of a MIMO system with relays," 2007 IEEE Inform. Theory Workshop on Information Theory for Wireless Networks, July 2007.

[8] M. Yuksel and E. Erkip, "Multiple-antenna cooperative wireless systems: a diversity-multiplexing tradeoff perspective," IEEE Trans. Inf. Theory, vol. 53, no. 10, pp. 3371-3393, Oct. 2007.

[9] Y. Fan, J. Thompson, A. Adinoyi, and H. Yanikomeroglu, "On the diversity-multiplexing tradeoff for multi-antenna multi-relay channels," in Proc. IEEE ICC 2007, pp. 5252-5256.

[10] N. C. Beaulieu and J. Hu, "Closed-form expression for the outage probability of decode-and-forward relaying in dissimilar Rayleigh fading channels," IEEE Commun. Lett., vol. 10, no. 12, pp. 813-815, Dec. 2006.

[11] G. Atia, M. Sharif, and V. Saligrama, "On optimal outage in relay channels with general fading distributions," IEEE Trans. Inf. Theory, vol. 53, no. 10, pp.3786-3797, Oct. 2007. 